\title{
The Weak Circular Repetition Threshold over Large Alphabets
}

\author{
Lucas Mol and Narad Rampersad* \\ Department of Mathematics and Statistics \\ The University of Winnipeg \\ 515 Portage Ave. \\ Winnipeg, MB, Canada \\ R3B 2E9 \\ \{l.mol, n.rampersad\}@uwinnipeg.ca
}

\begin{abstract}
The repetition threshold for words on $n$ letters, denoted $\mathrm{RT}(n)$, is the infimum of the set of all $r$ such that there are arbitrarily long $r$-free words over $n$ letters. A repetition threshold for circular words on $n$ letters can be defined in three natural ways, which gives rise to the weak, intermediate, and strong circular repetition thresholds for $n$ letters, denoted $\operatorname{CRT}_{\mathrm{W}}(n), \operatorname{CRT}_{\mathrm{I}}(n)$, and $\operatorname{CRT}_{\mathrm{S}}(n)$, respectively. Currie and the present authors conjectured that $\operatorname{CRT}_{\mathrm{I}}(n)=\mathrm{CRT}_{\mathrm{W}}(n)=\mathrm{RT}(n)$ for all $n \geq 4$. We prove that $\operatorname{CRT}_{\mathrm{W}}(n)=\mathrm{RT}(n)$ for all $n \geq 45$, which confirms a weak version of this conjecture for all but finitely many values of $n$.

MSC 2010: 68R15 (primary), 05C15 (secondary)

Keywords: repetition threshold; circular repetition threshold; repetition threshold for graphs; Dejean's conjecture; Dejean's theorem; nonrepetitive colouring
\end{abstract}

${ }^{*}$ The work of Narad Rampersad is supported by the Natural Sciences and Engineering Research Council of Canada (NSERC), [funding reference number 2019-04111]. 


\section{Introduction}

Throughout, we use standard definitions and notations from combinatorics on words (see [16]). The word $u$ is a factor of the word $w$ if we can write $w=x u y$ for some (possibly empty) words $x, y$. If at least one of $x, y$ is nonempty, then we say that $u$ is a proper factor of $w$. For a set of words $L$, the word $u$ is a factor of $L$ if $u$ is a factor of some word in $L$.

Let $w=w_{1} w_{2} \cdots w_{k}$ be a word, where the $w_{i}$ 's are letters. A positive integer $p$ is a period of $w$ if $w_{i+p}=w_{i}$ for all $1 \leq i \leq k-p$. In this case, we say that $|w| / p$ is an exponent of $w$, and the largest such number is called the exponent of $w$. For a real number $r>1$, a finite or infinite word $w$ is called $r$-free $\left(r^{+}\right.$-free) if $w$ contains no factors of exponent greater than or equal to $r$ (strictly greater than $r$, respectively).

Throughout, for every positive integer $n$, let $A_{n}$ denote the $n$-letter alphabet $\{1,2, \ldots, \mathrm{n}\}$. For every $n \geq 2$, the repetition threshold for $n$ letters, denoted $\operatorname{RT}(n)$, is defined by

$$
\mathrm{RT}(n)=\inf \left\{r>1: \text { there are arbitrarily long } r \text {-free words over } A_{n}\right\} .
$$

Essentially, the repetition threshold describes the border between avoidable and unavoidable repetitions in words over an alphabet of $n$ letters. The repetition threshold was first defined by Dejean [13]. Her 1972 conjecture on the values of $\operatorname{RT}(n)$ has now been confirmed through the work of many authors $5,7,9,13,18,19,22,23]$ :

$$
\operatorname{RT}(n)= \begin{cases}2, & \text { if } n=2 \\ 7 / 4, & \text { if } n=3 \\ 7 / 5, & \text { if } n=4 \\ n /(n-1), & \text { if } n \geq 5\end{cases}
$$

The last cases of Dejean's conjecture were confirmed in 2011 by Currie and the second author [9], and independently by Rao [23]. However, probably the most important contribution was made by Carpi [5], who confirmed the conjecture for all but finitely many values of $n$.

Here, we are interested in the notion of a repetition threshold for circular words on $n$ letters. Two (linear) words $x$ and $y$ are said to be conjugates if there are words $u$ and $v$ such that $x=u v$ and $y=v u$. The conjugates of a word $w$ can be obtained by rotating the letters of $w$ cyclically. For a word $w$, 
the circular word $\langle w\rangle$ is the set of all conjugates of $w$. Intuitively, one can think of a circular word as being obtained from a linear word by linking the ends, giving a cyclic sequence of letters. A circumnavigation of the circular word $\langle w\rangle$ is a word of the form $a v a$, where $a$ is a letter and $a v$ is a conjugate of $w$.

By the definition of a factor of a set of words, a word is a factor of a circular word $\langle w\rangle$ if and only if it is a factor of some conjugate of $w$. As for linear words, a circular word is $r$-free $\left(r^{+}\right.$-free $)$if it has no factors of exponent greater than or equal to $r$ (strictly greater than $r$, respectively).

While the factors of an $r$-free circular word must be $r$-free as linear words, they need not necessarily be $r$-free when taken as circular words. For example, the 2-free circular word $\langle 012021\rangle$ contains the factor 0120, and the circular word $\langle 0120\rangle$ contains the square factor 00. This means that several equivalent definitions of the repetition threshold $\mathrm{RT}(n)$ give rise to distinct notions of a repetition threshold for circular words.

In this paper, we will be most interested in the weak circular repetition threshold for $n$ letters, denoted $\operatorname{CRT}_{\mathrm{W}}(n)$, and defined by

$\mathrm{CRT}_{\mathrm{W}}(n)=\inf \left\{r>1\right.$ : there are arbitrarily long $r$-free circular words over $\left.A_{n}\right\}$.

The intermediate circular repetition threshold for $n$ letters, $\operatorname{denoted} \mathrm{CRT}_{\mathrm{I}}(n)$, is defined by

$\operatorname{CRT}_{\mathrm{I}}(n)=\inf \left\{r>1:\right.$ there are $r$-free circular words of every sufficiently large length over $\left.A_{n}\right\}$,

and the strong circular repetition threshold for $n$ letters, denoted $\mathrm{CRT}_{\mathrm{S}}(n)$, is defined by

$$
\mathrm{CRT}_{\mathrm{S}}(n)=\inf \left\{r>1: \text { there are } r \text {-free circular words of every length over } A_{n}\right\} \text {. }
$$

Evidently, we have

$$
\mathrm{RT}(n) \leq \operatorname{CRT}_{\mathrm{W}}(n) \leq \operatorname{CRT}_{\mathrm{I}}(n) \leq \operatorname{CRT}_{\mathrm{S}}(n)
$$

for all $n \geq 2$. Table 1 contains all of the confirmed and conjectured values of $\mathrm{RT}(n), \mathrm{CRT}_{\mathrm{W}}(n), \mathrm{CRT}_{\mathrm{I}}(n)$, and $\mathrm{CRT}_{\mathrm{S}}(n)$. Note in particular that

$$
\operatorname{CRT}_{\mathrm{W}}(2)<\operatorname{CRT}_{\mathrm{I}}(2)<\operatorname{CRT}_{\mathrm{S}}(2)
$$

which shows that the three notions of circular repetition threshold are not equivalent. 


\begin{tabular}{r|cccc}
$n$ & 2 & 3 & 4 & $n \geq 5$ \\
\hline $\operatorname{RT}(n)$ & $\mathbf{2}$ & $\frac{\mathbf{7}}{\mathbf{4}}$ & $\frac{\mathbf{7}}{\mathbf{5}}$ & $\frac{\boldsymbol{n}}{\boldsymbol{n - 1}}$ \\
$\mathrm{CRT}_{\mathrm{W}}(n)$ & $\mathbf{2}$ & $\frac{\mathbf{7}}{\mathbf{4}}$ & $\frac{7}{5}$ & $\frac{n}{n-1}$ \\
$\mathrm{CRT}_{\mathrm{I}}(n)$ & $\mathbf{7}$ & $\frac{\mathbf{7}}{\mathbf{4}}$ & $\frac{7}{5}$ & $\frac{n}{n-1}$ \\
$\mathrm{CRT}_{\mathrm{S}}(n)$ & $\mathbf{5}$ & $\mathbf{2}$ & $\frac{\mathbf{3}}{\mathbf{2}}$ & $\frac{\lceil\boldsymbol{n} / \mathbf{2}\rceil+\mathbf{1}}{\lceil\boldsymbol{n} / \mathbf{2}\rceil}$
\end{tabular}

Table 1: The confirmed and conjectured values of the weak, intermediate, and strong circular repetition thresholds. Confirmed values are in bold font, while conjectured values are in normal font (and coloured red).

Through the work of several authors [1, 10, 12, 15, 24], all values of the strong circular repetition threshold are known:

$$
\mathrm{CRT}_{\mathrm{S}}(n)= \begin{cases}5 / 2, & \text { if } n=2 \\ 2, & \text { if } n=3 \\ \frac{\lceil n / 2\rceil+1}{\lceil n / 2\rceil}, & \text { if } n \geq 4\end{cases}
$$

The values of the weak and intermediate circular repetition thresholds are only known exactly for $n=2$ and $n=3$ :

- $\operatorname{CRT}_{\mathrm{W}}(2)=2($ Thue $[4])$;

- $\operatorname{CRT}_{\mathrm{I}}(2)=7 / 3($ Aberkane and Currie [2], Shur [24]); and

- $\operatorname{CRT}_{\mathrm{I}}(3)=\operatorname{CRT}_{\mathrm{W}}(3)=7 / 4$ (Shur [24], Currie et al. [10]).

From (1) and the known values of $\operatorname{RT}(n)$ and $\operatorname{CRT}_{\mathrm{S}}(n)$, we have

$$
\frac{n}{n-1} \leq \operatorname{CRT}_{\mathrm{W}}(n) \leq \operatorname{CRT}_{\mathrm{I}}(n) \leq \frac{\lceil n / 2\rceil+1}{\lceil n / 2\rceil}
$$

for every $n \geq 5$. These are currently the best known bounds on both $\mathrm{CRT}_{\mathrm{W}}(n)$ and $\operatorname{CRT}_{\mathrm{I}}(n)$ when $n \geq 5$. Currie and the present authors 10 recently made the following conjecture, which strengthens the second statement of an older conjecture of Shur [24, Conjecture 1].

Conjecture 1. For every $n \geq 4$, we have $\operatorname{CRT}_{\mathrm{I}}(n)=\mathrm{CRT}_{\mathrm{W}}(n)=\operatorname{RT}(n)$. 
Here, we prove a weak version (pun intended) of Conjecture 1 for all but finitely many values of $n$.

Theorem 2. For every $n \geq 45$, we have $\operatorname{CRT}_{\mathrm{W}}(n)=\mathrm{RT}(n)=n /(n-1)$.

The layout of the remainder of the paper is as follows. In Section 2, we summarize the work of Carpi $[5]$ in confirming all but finitely many cases of Dejean's conjecture. In Section 3, we establish Theorem 2 with a construction that relies heavily on the work of Carpi. We conclude with a discussion of some related notions of repetition threshold for classes of graphs.

We note that the work of Carpi [5] that we rely on in this paper was also instrumental in the recent proof by Currie and the present authors 11 that for every $n \geq 27$, the number of $n /(n-1)^{+}$-free words of length $k$ over $n$ letters grows exponentially in $k$. This speaks to the strength of Carpi's results.

\section{Carpi's reduction to $\psi_{n}$-kernel repetitions}

In this section, let $n \geq 2$ be a fixed integer. Pansiot [22] was first to observe that if a word over the alphabet $A_{n}$ is $(n-1) /(n-2)$-free, then it can be encoded by a word over the binary alphabet $B=\{0,1\}$. For consistency, we use the notation of Carpi [5] to describe this encoding. Let $\mathbb{S}_{n}$ denote the symmetric group on $A_{n}$, and define the morphism $\varphi_{n}: B^{*} \rightarrow \mathbb{S}_{n}$ by

$$
\begin{aligned}
& \varphi_{n}(0)=\left(\begin{array}{llll}
1 & 2 & \cdots & \mathrm{n}-1
\end{array}\right) ; \text { and } \\
& \varphi_{n}(1)=\left(\begin{array}{llll}
1 & 2 & \cdots & \mathrm{n}
\end{array}\right) .
\end{aligned}
$$

Now define the map $\gamma_{n}: B^{*} \rightarrow A_{n}^{*}$ by

$$
\gamma_{n}\left(b_{1} b_{2} \cdots b_{k}\right)=a_{1} a_{2} \cdots a_{k}
$$

where

$$
a_{i} \varphi_{n}\left(b_{1} b_{2} \cdots b_{i}\right)=1
$$

for all $1 \leq i \leq k$. To be precise, Pansiot proved that if a word $\alpha \in A_{n}^{*}$ is $(n-1) /(n-2)$-free, then $\alpha$ can be obtained from a word of the form $\gamma_{n}(u)$, where $u \in B^{*}$, by renaming the letters.

Let $u \in B^{*}$, and let $\alpha=\gamma_{n}(u)$. Pansiot showed that if $\alpha$ has a factor of exponent greater than $n /(n-1)$, then either the word $\alpha$ itself contains a 
short repetition, or the binary word $u$ contains a kernel repetition (see 22] for details). Carpi reformulated this statement so that both types of forbidden factors appear in the binary word $u$. Let $k \in\{1,2, \ldots, n-1\}$, and let $v \in B^{+}$. Then $v$ is called a $k$-stabilizing word (of order $n$ ) if $\varphi_{n}(v)$ fixes the points $1,2, \ldots, \mathrm{k}$. Let $\operatorname{Stab}_{n}(k)$ denote the set of $k$-stabilizing words of order $n$. The word $v$ is called a kernel repetition (of order $n$ ) if it has period $p$ and a factor $v^{\prime}$ of length $p$ such that $v^{\prime} \in \operatorname{ker}\left(\varphi_{n}\right)$ and $|v|>\frac{n p}{n-1}-(n-1)$. Carpi's reformulation of Pansiot's result is the following.

Proposition 3 (Carpi [5, Proposition 3.2]). Let $u \in B^{*}$. If a factor of $\gamma_{n}(u)$ has exponent larger than $n /(n-1)$, then $u$ has a factor $v$ satisfying one of the following conditions:

(i) $v \in \operatorname{Stab}_{n}(k)$ and $0<|v|<k(n-1)$ for some $1 \leq k \leq n-1$; or

(ii) $v$ is a kernel repetition of order $n$.

Now assume that $n \geq 9$, and define $m=\lfloor(n-3) / 6\rfloor$ and $\ell=\lfloor n / 2\rfloor$. Carpi [5] defines an $(n-1)(\ell+1)$-uniform morphism $f_{n}: A_{m}^{*} \rightarrow B^{*}$ with the following extraordinary property.

Proposition 4 (Carpi [5, Proposition 7.3]). Suppose that $n \geq 27$, and let $w \in A_{m}^{*}$. Then for every $k \in\{1,2, \ldots, n-1\}$, the word $f_{n}(w)$ contains no $k$-stabilizing word of length smaller than $k(n-1)$.

We note that Proposition 4 was proven by Carpi $[5]$ in the case that $n \geq 30$ in a computation-free manner. The improvement to $n \geq 27$ stated here was achieved later by Currie and the second author [7], using lemmas of Carpi [5] along with a significant computer check.

Proposition 4 says that for every word $w \in A_{m}^{*}$, no factor of $f_{n}(w)$ satisfies condition (i) of Proposition 3. Thus, we need only worry about factors satisfying condition (ii) of Proposition 3, i.e., kernel repetitions. To this end, define the morphism $\psi_{n}: A_{m}^{*} \rightarrow \mathbb{S}_{n}$ by $\psi_{n}(v)=\varphi_{n}\left(f_{n}(v)\right)$ for all $v \in A_{m}^{*}$. A word $v \in A_{m}^{*}$ is called a $\psi_{n}$-kernel repetition if it has a period $q$ and a factor $v^{\prime}$ of length $q$ such that $v^{\prime} \in \operatorname{ker}\left(\psi_{n}\right)$ and $(n-1)(|v|+1) \geq n q-3$. Carpi established the following result.

Proposition 5 (Carpi [5, Proposition 8.2]). Let $w \in A_{m}^{*}$. If a factor of $f_{n}(w)$ is a kernel repetition, then a proper factor of $w$ is a $\psi_{n}$-kernel repetition. 
Remark. The word "proper" in the statement of Proposition 5 was not in the original statement of Carpi [5, Proposition 8.2], but it is easily verified that Carpi's proof actually proves this stronger statement, which will be necessary for our work.

In other words, if no proper factor of $w \in A_{m}^{*}$ is a $\psi_{n}$-kernel repetition, then no factor of $f_{n}(w)$ satisfies condition (ii) of Proposition 3 . Finally, we note that the morphism $f_{n}$ is defined in such a way that the kernel of $\psi_{n}$ has a very simple structure.

Lemma 6 (Carpi [5, Lemma 9.1]). If $v \in A_{m}^{*}$, then $v \in \operatorname{ker}\left(\psi_{n}\right)$ if and only if 4 divides $|v|_{a}$ for every letter $a \in A_{m}$.

\section{Constructing $n /(n-1)^{+}$-free circular words over $n$ letters}

In this section, let $n \geq 27$ be a fixed integer, and let $m=\lfloor(n-3) / 6\rfloor$ and $\ell=\lfloor n / 2\rfloor$, as in the previous section. Finally, define $M=4^{m-2}$. Since $n \geq 27$, we have $m \geq 4$ and $M \geq 16$.

In order to prove Theorem 2 , we will construct an $n /(n-1)^{+}$-free circular word of length $M(n-1)(\ell+1) t$ over $A_{n}$ for every integer $t \geq 1$. We first show that we can restrict our attention to words over the smaller alphabet $A_{m}$, just as Carpi did for linear words. We begin with an analogue of Proposition 3 for circular words.

Lemma 7. Let $u \in B^{*} \cap \operatorname{ker}\left(\varphi_{n}\right)$. If a factor of the circular word $\left\langle\gamma_{n}(u)\right\rangle$ has exponent larger than $n /(n-1)$, then the circular word $\langle u\rangle$ has a factor $v$ satisfying one of the following conditions:

(i) $v \in \operatorname{Stab}_{n}(k)$ and $0<|v|<k(n-1)$ for some $1 \leq k \leq n-1$; or

(ii) $v$ is a kernel repetition of order $n$.

Proof. Let $u=u_{1} u_{2} \cdots u_{s}$, where $u_{i} \in B$ for all $i \in\{1, \ldots, s\}$. Let $\gamma_{n}(u)=$ $a_{1} a_{2} \ldots a_{s}$, where $a_{i} \in A_{n}$ for all $i \in\{1, \ldots, s\}$. First, we claim that for every conjugate $u^{\prime}=u_{j} \cdots u_{s} u_{1} \cdots u_{j-1}$ of $u$, the word $\gamma\left(u^{\prime}\right)$ is equal, up to a permutation of the letters, to the corresponding conjugate $a_{j} \cdots a_{s} a_{1} \cdots a_{j-1}$ 
of $\gamma_{n}(u)$. It suffices to show that $\gamma_{n}\left(u_{2} u_{3} \cdots u_{s} u_{1}\right)$ is equal to $a_{2} a_{3} \cdots a_{k} a_{1}$, up to a permutation of the letters. By definition of $\gamma_{n}$, we have

$$
a_{i} \varphi_{n}\left(u_{1} u_{2} \cdots u_{i}\right)=1
$$

for all $i \in\{1, \ldots, s\}$. Let $\gamma_{n}\left(u_{2} u_{3} \cdots u_{s} u_{1}\right)=b_{2} b_{3} \cdots b_{s} b_{1}$. Again, by definition of $\gamma_{n}$, we have

$$
b_{i}\left[\varphi_{n}\left(u_{1}\right)\right]^{-1} \varphi_{n}\left(u_{1} u_{2} \ldots u_{i}\right)=1
$$

for all $i \in\{2, \ldots, s\}$. Finally, using the definition of $\gamma_{n}$ and the fact that $u \in \operatorname{ker}\left(\varphi_{n}\right)$, we have

$$
b_{1}\left[\varphi_{n}\left(u_{1}\right)\right]^{-1} \varphi_{n}\left(u_{1}\right)=b_{1}\left[\varphi_{n}\left(u_{1}\right)\right]^{-1} \varphi_{n}\left(u_{1} u_{2} \ldots u_{s} u_{1}\right)=1 .
$$

Thus, we see that $b_{i}=a_{i} \varphi_{n}\left(u_{1}\right)$ for all $i \in\{1, \ldots, s\}$, and this completes the proof of the claim.

Suppose now that a factor $w$ of the circular word $\left\langle\gamma_{n}(u)\right\rangle$ has exponent larger than $n /(n-1)$. Then, up to permutation of the letters, the word $w$ is a factor of $\gamma_{n}\left(u^{\prime}\right)$ for some conjugate $u^{\prime}$ of $u$. By Lemma 3, the word $u^{\prime}$ contains a factor $v$ satisfying either condition (i) or condition (ii). Since $u^{\prime}$ is a conjugate of $u$, the word $v$ is a factor of $\langle u\rangle$, and this completes the proof.

The next lemma is an analogue of Proposition 5 for circular words.

Lemma 8. Let $w \in A_{m}^{*} \cap \operatorname{ker}\left(\psi_{n}\right)$. If the circular word $\langle w\rangle$ contains no $\psi_{n}$-kernel repetitions, then the circular word $\left\langle\gamma_{n}\left(f_{n}(w)\right)\right\rangle$ is $n /(n-1)^{+}$-free.

Proof. Suppose, towards a contradiction, that $\langle w\rangle$ contains no $\psi_{n}$-kernel repetitions, and that $\left\langle\gamma_{n}\left(f_{n}(w)\right)\right\rangle$ contains a factor of exponent greater than $n /(n-1)$. Since $w \in \operatorname{ker}\left(\psi_{n}\right)$, we have $f_{n}(w) \in \operatorname{ker}\left(\varphi_{n}\right)$. Thus, by Lemma 7 , some factor $v$ of $\left\langle f_{n}(w)\right\rangle$ satisfies either condition (i) or condition (ii) of Lemma 7. Now $v$ must be a factor of $f_{n}\left(w^{\prime}\right)$ for some circumnavigation $w^{\prime}$ of $w$ (i.e., we have $w^{\prime}=a w^{\prime \prime} a$, where $a \in A_{m}$ and $a w^{\prime \prime}$ is a conjugate of $w$ ). By Proposition 4, for every $1 \leq k \leq n-1$, the word $f_{n}\left(w^{\prime}\right)$ contains no $k$-stabilizing words of length less than $k(n-1)$. So it must be the case that $v$ is a kernel repetition of order $n$. Then by Proposition 5, some proper factor of $w^{\prime}$ must be a $\psi_{n}$-kernel repetition. Since every proper factor of the circumnavigation $w^{\prime}$ is a factor of the circular word $\langle w\rangle$, this is a contradiction. So we conclude that the circular word $\left\langle\gamma_{n}\left(f_{n}(w)\right)\right\rangle$ is $n /(n-1)^{+}$-free. 


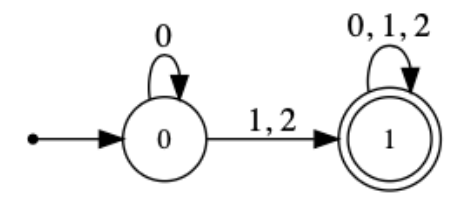

Figure 1: The automaton accepting those $(k)_{3}$ such that the word $\beta$ has a factor of length $k$ that begins and ends in the letter 2 .

By Lemma 8, in order to construct an $n /(n-1)^{+}$-free circular word of length $M(n-1)(\ell+1) t$ over $A_{n}$, it suffices to construct a word of length $M t$ over $A_{m}$ that lies in $\operatorname{ker}\left(\psi_{n}\right)$ and contains no $\psi_{n}$-kernel repetitions. Our construction of such a word uses many ideas of Carpi [5, Section 9], and we recommend that the reader reviews this section before proceeding.

Following Carpi, define $\beta=\left(b_{i}\right)_{i \geq 1}$, where

$$
b_{i}=\left\{\begin{array}{lll}
1, & \text { if } i \equiv 1 & (\bmod 3) \\
2, & \text { if } i \equiv 2 & (\bmod 3) \\
b_{i / 3}, & \text { if } i \equiv 0 & (\bmod 3)
\end{array}\right.
$$

We note that $\beta$ can also be defined (c.f. [6. Example 4]) as the fixed point of the morphism $\tau: A_{2}^{*} \rightarrow A_{2}^{*}$ defined by

$$
\begin{aligned}
& 1 \mapsto 121 \\
& 2 \mapsto 122 .
\end{aligned}
$$

We need two lemmas concerning the factors of $\beta$.

Lemma 9. For every $k \geq 1$, the word $\beta$ has a factor of length $k$ that begins and ends in the letter 2.

Proof. The statement can be verified by a case-based proof depending on the value of $k \bmod 3$, or by using the automatic theorem proving software Walnut [20]. We describe the latter approach. After saving the automaton generating the fixed point of $\tau$ in the Word Automata Library folder as B.txt, we use the predicate

eval BeginsAndEndsInTwo "?msd_3 ( $\mathrm{k}>=1$ \& (Ei (B $[i]=@ 2$ \& $B[i+k-1]=@ 2)))$ ":

The automaton for this predicate is illustrated in Figure 1. The automaton clearly accepts all $(k)_{3}$ such that $k \geq 1$. 
Lemma 10 (Carpi [5, Lemma 9.2]). Let $u$ be a factor of $\beta$ with period $q$. For every $k \geq 0$, if $|u| \geq q+3^{k}$, then $3^{k}$ divides $q$.

For every $t \geq 1$, we define a set of words $\Lambda_{t} \subseteq A_{m}^{*}$, all of length $M t$. We define $\Lambda_{t}$ by $x_{0} x_{1} \cdots x_{M t-1} \in \Lambda_{t}$ if and only if $x_{i}=\max \{a \in$ $A_{m}: 4^{a-3}$ divides $\left.i\right\}$ whenever $i \equiv 0(\bmod 4)$, and $x_{i} \in\{1,2,3\}$ whenever $i \not \equiv 0(\bmod 4)$.

We prove two lemmas concerning the words in $\Lambda_{t}$. The proof of the first lemma uses the main idea from Carpi's proof of [5, Lemma 9.3].

Lemma 11. Let $x \in \Lambda_{t}$, and let $v$ be a factor of the circular word $\langle x\rangle$. If $v \in \operatorname{ker}\left(\psi_{n}\right)$, then $M$ divides $|v|$.

Proof. The statement is trivially true if $v=\varepsilon$, so assume $|v|>0$. Set $|v|=4^{b} c$, where $4^{b}$ is the maximal power of 4 dividing $|v|$. Suppose, towards a contradiction, that $b \leq m-3$. Since $v \in \operatorname{ker}\left(\psi_{n}\right)$, by Lemma 6 , we see that 4 divides $|v|$, meaning $b \geq 1$.

Write $x=x_{0} x_{1} \cdots x_{M t-1}$. Then we have $v=x_{i} x_{i+1} \cdots x_{i+4^{b} c-1}$ for some $i \geq 1$, with indices taken modulo $M t$. Since $M t$ is divisible by $M=4^{m-2}$ and $b<m-2$, for all $j \geq 0$ and $k \in\{1,2, \ldots, b\}$, we see that $4^{k}$ divides $j$ if and only if $4^{k}$ divides $j \bmod M t$. Since $b \geq 1$, we have $b+3 \geq 4$, and hence $x_{j} \geq b+3$ implies $j \equiv 0(\bmod 4)$. Thus, by definition, for any $j \in\left\{i, i+1, \ldots, i+4^{b} c-1\right\}$, we have $x_{j} \geq b+3$ if and only if $4^{b}$ divides $j$. Thus, we have that the sum $\sum_{a=b+3}^{m}|v|_{a}$ is exactly the number of integers in the set $\left\{i, i+1, \ldots, i+4^{b} c-1\right\}$ that are divisible by $4^{b}$, which is exactly $c$. Since $v \in \operatorname{ker}\left(\psi_{n}\right)$, by Lemma 6, we conclude that 4 divides $c$, contradicting the maximality of $b$.

Lemma 12. Let $x \in \Lambda_{t}$. For every letter $a \in\{4,5, \ldots, \mathrm{m}\}$, the number $|x|_{a}$ is a multiple of 4 .

Proof. Write $x=x_{0} x_{1} \ldots x_{M t-1}$, and let $a \in\{4,5, \ldots, \mathrm{m}\}$. Since $x \in \Lambda_{t}$, we have $x_{i} \geq a$ if and only if $4^{a-3}$ divides $i$. Since $|x|=M t=4^{m-2} t$, we have

$$
\sum_{b=a}^{m}|x|_{b}=M t / 4^{a-3}=4^{1+m-a} t .
$$

Since $m-a+1 \geq 1$, we see that 4 divides $\sum_{b=a}^{m}|x|_{b}$. The fact that 4 divides $|x|_{a}$ now follows by a straightforward inductive argument. 
We are now ready to construct a circular word of length $M t$ over $A_{m}$ that belongs to $\operatorname{ker}\left(\psi_{n}\right)$ and contains no $\psi_{n}$-kernel repetitions. The proof of the next proposition uses some arguments that were first used by Carpi [5, Proposition 9.4].

Proposition 13. Suppose that $n \geq 45$. For every integer $t \geq 1$, there is a word $w \in A_{m}^{*} \cap \operatorname{ker}\left(\psi_{n}\right)$ of length $M t$ such that the circular word $\langle w\rangle$ contains no $\psi_{n}$-kernel repetitions.

Proof. Fix $t \geq 1$. Since $n \geq 45$, we have $m \geq 7$ and $M=4^{m-2} \geq 4^{5}$. We use these facts frequently. Let $u$ be a factor of $\beta$ of length $M t / 4$ that begins and ends in the letter 2; such a factor is guaranteed to exist by Lemma 9 . Let $\sigma: A_{2}^{*} \rightarrow\{1,3\}^{*}$ be the morphism defined by

$$
\begin{aligned}
1 & \mapsto 1 \\
2 & \mapsto 3 .
\end{aligned}
$$

Write $u \sigma(u)=u_{1} u_{2} \ldots u_{M t / 2}$.

Let $s=4-\left(|u|_{2} \bmod 4\right)$, and define $v=v_{1} v_{2} \cdots v_{M t / 4}$ by

$$
v_{i}= \begin{cases}3, & \text { if } 1 \leq i \leq s \\ 2, & \text { if } s+1 \leq i \leq 2 s \\ 1, & \text { if } 2 s \leq i \leq M t / 4\end{cases}
$$

Finally, define $w=w_{0} w_{1} \cdots w_{M t-1}$ by

$$
w_{i}= \begin{cases}\max \left\{a \in A_{m}: 4^{a-3} \text { divides } i\right\}, & \text { if } i \equiv 0(\bmod 4) ; \\ v_{(i+2) / 4}, & \text { if } i \equiv 2(\bmod 4) ; \\ u_{(i+1) / 2}, & \text { if } i \text { is odd. }\end{cases}
$$

Evidently, we have $w \in \Lambda_{t}$. We will show that $w \in \operatorname{ker}\left(\psi_{n}\right)$, and that the circular word $\langle w\rangle$ contains no $\psi_{n}$-kernel repetitions, thus proving the proposition statement.

First, we show that $w \in \operatorname{ker}\left(\psi_{n}\right)$. By Lemma 5 , it suffices to show that 4 divides $|w|_{a}$ for all $a \in\{1,2, \ldots, \mathrm{m}\}$. Since $w \in \Lambda_{t}$, by Lemma 12 , we have that 4 divides $|w|_{a}$ for all $a \in\{4,5, \ldots, \mathrm{m}\}$. Next, note that

$$
|w|_{2}=|u|_{2}+s=|u|_{2}+4-\left(|u|_{2} \bmod 4\right),
$$


which is clearly divisible by 4 . Since

$$
|w|_{3}=|\sigma(u)|_{3}+s=|u|_{2}+s=|w|_{2}
$$

we see that 4 divides $|w|_{3}$ as well. Finally, we have

$$
|w|_{1}=|w|-\sum_{b=2}^{m}|w|_{b}=M t-\sum_{b=2}^{m}|w|_{b} .
$$

Since 4 divides both $M$ and $|w|_{b}$ for all $b \geq 2$, we conclude that 4 divides $|w|_{1}$.

It remains to show that the circular word $\langle w\rangle$ contains no $\psi_{n}$-kernel repetitions. Suppose towards a contradiction that some factor $X$ of $\langle w\rangle$ is a $\psi_{n}$-kernel repetition of period $q$. Then by definition, we have

$$
n q-3 \leq(n-1)(|X|+1) .
$$

Since $m=\lfloor(n-3) / 6\rfloor$, we must also have $n \leq 6 m+8$.

First of all, consider the case $|X| \leq q+5$. Applying this inequality to the right side of (2) and then rearranging, we obtain

$$
q \leq 6 n-3
$$

By Lemma 11, we must have $q \geq M=4^{m-2}$. Together with the fact that $n \leq 6 m+8$, this gives

$$
4^{m-2} \leq 36 m+45 .
$$

But this last inequality implies $m \leq 6$, a contradiction.

So we may assume that $|X| \geq q+6$. Then one has $q+2 \cdot 3^{k} \leq|X|<q+2$. $3^{k+1}$ for some integer $k \geq 1$. Using (2) and the inequality $|X|+1 \leq q+2 \cdot 3^{k+1}$, we obtain

$$
q \leq 2(n-1) 3^{k+1}+3 \leq 3^{k}(36 m+45) .
$$

Deleting the letters of even index in $X$, we obtain a factor $x$ of the circular word $\langle u \sigma(u)\rangle$. By Lemma 11, we know that $4^{m-2}$ divides $q$, so $q$ is certainly divisible by 2 , and $x$ has period $q / 2$. Since $|X| \geq q+2 \cdot 3^{k}$, we have

$$
|x| \geq\left\lfloor\frac{|X|}{2}\right\rfloor \geq q / 2+3^{k} \geq q / 2+3 \text {. }
$$


Write $x=x^{\prime} y=y x^{\prime \prime}$, where $\left|x^{\prime}\right|=\left|x^{\prime \prime}\right|=q / 2$. From (4), we see that $|y| \geq 3$.

By construction, we may write $u=2 u^{\prime} 2$ and $\sigma(u)=3 u^{\prime \prime} 3$, where $u^{\prime} \in$ $\{1,2\}^{*}$ and $u^{\prime \prime} \in\{1,3\}^{*}$. So we have

$$
u \sigma(u)=2 u^{\prime} 23 u^{\prime \prime} 3
$$

Thus, in the circular word $\langle u \sigma(u)\rangle$, the factors 23 and 32 both appear exactly once. Further, every factor of length 3 of $u$ contains a 2, and every factor of length 3 of $\sigma(u)$ contains a 3. Therefore, either every appearance of $y$ in $\langle u \sigma(u)\rangle$ is contained entirely in $u$, or every appearance of $y$ in $\langle u \sigma(u)\rangle$ is contained entirely in $\sigma(u)$. Without loss of generality, assume that every appearance of $y$ in $\langle u \sigma(u)\rangle$ is contained entirely in $u$. Now we consider two cases.

Case 1: The factor $x$ is contained entirely in $u$.

Then $x$ is a factor of $\beta$ with period $q / 2$. By Lemma 11, we know that $4^{m-2}$ divides $q$. Recall from (4) that $|x| \geq q / 2+3^{k}$. Thus, by Lemma 10, we have that $3^{k}$ divides $q / 2$. Therefore, we have $q \geq 4^{m-2} 3^{k}$. Together with (3), this gives

$$
4^{m-2} \leq 36 m+45
$$

But we have already seen that this inequality implies $m \leq 6$, a contradiction.

Case 2: The factor $x$ contains the entire word $\sigma(u)$ as a factor.

Then we can write $x=y z y$, where $|y z|=q / 2$, and $z$ contains the entire word $\sigma(u)$. Let $y z y z^{\prime}$ be a conjugate of $u \sigma(u)$. See Figure 2 for an illustration of the circular word $\langle u \sigma(u)\rangle$.

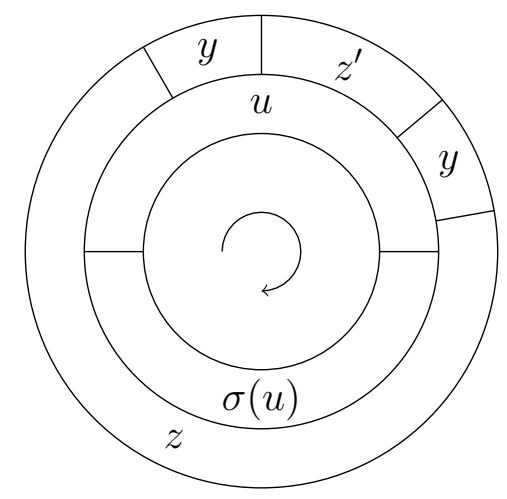

Figure 2: The circular word $\langle u \sigma(u)\rangle$ in Case 2 of the proof of Proposition 13 . 
Now let $Y Z Y Z^{\prime}$ be the conjugate of $w$ corresponding to $y z y z^{\prime}$, i.e., we have that $Y Z$ is the length $q$ prefix of $X,|Y|=2|y|,|Z|=2|z|$, and $\left|Z^{\prime}\right|=$ $2\left|z^{\prime}\right|$. We claim that $Y Z^{\prime} Y$ is a $\psi_{n}$-kernel repetition with period $\left|Y Z^{\prime}\right|$. First of all, note that since $y z^{\prime} y$ is a factor of $u$, while $z$ contains all of $\sigma(u)$, we have $|Z|>\left|Z^{\prime}\right|$. Next, note that both $Y Z \in \operatorname{ker}\left(\psi_{n}\right)$ and $Y Z Y Z^{\prime} \in \operatorname{ker}\left(\psi_{n}\right)$. Now let $a \in A_{m}$. By Lemma 5, both $|Y Z|_{a}$ and $\left|Y Z Y Z^{\prime}\right|_{a}$ are multiples of 4. It follows that

$$
\left|Y Z^{\prime}\right|_{a}=\left|Y Z Y Z^{\prime}\right|_{a}-|Y Z|_{a}
$$

is also a multiple of 4 , and hence $Y Z^{\prime} \in \operatorname{ker}\left(\psi_{n}\right)$. By the definition of $\psi_{n^{-}}$ kernel repetition, we have

$$
(n-1)(|Y Z Y|+1) \geq n \cdot|Y Z|-3 .
$$

Rearranging the above inequality, and then using the fact that $|Z|>\left|Z^{\prime}\right|$, we have

$$
(n-2)|Y|+n+2 \geq|Z|>\left|Z^{\prime}\right|,
$$

which implies that $Y Z^{\prime} Y$ is also a $\psi_{n}$-kernel repetition. But this is impossible by Case 1 .

Finally, Theorem 2 follows directly from the next result.

Proposition 14. Suppose that $n \geq 45$. For every integer $t \geq 1$, there is a word $W \in A_{n}^{*}$ of length $M(n-1)(\ell+1) t$ such that the circular word $\langle W\rangle$ is $n /(n-1)^{+}$-free.

Proof. Fix $t \geq 1$. By Proposition 13, there is a word $w \in A_{m}^{*} \cap \operatorname{ker}\left(\psi_{n}\right)$ of length $M t$ such that the circular word $\langle w\rangle$ contains no $\psi_{n}$-kernel repetitions. Since $f_{n}$ is $(n-1)(\ell+1)$-uniform, and $\gamma_{n}$ preserves length, we have $\left|\gamma_{n}\left(f_{n}(w)\right)\right|=M(n-1)(\ell+1) t$. By Lemma 8 , the circular word $\left\langle\gamma_{n}\left(f_{n}(w)\right)\right\rangle$ is $n /(n-1)^{+}$-free.

\section{Conclusion}

We have shown that $\operatorname{CRT}_{\mathrm{W}}(n)=\mathrm{RT}(n)=n /(n-1)$ for all $n \geq 45$. The conjecture that $\operatorname{CRT}_{\mathrm{W}}(n)=\mathrm{RT}(n)$ remains open for all $4 \leq n \leq 44$, and the stronger conjecture that $\mathrm{CRT}_{\mathrm{I}}(n)=\mathrm{RT}(n)$ remains open for all $n \geq 4$.

To conclude, we will place the notion of weak circular repetition threshold in a broader context, and discuss a more general problem. Let $G$ be a graph, 
and let $f: V(G) \rightarrow A_{n}$ be an $n$-colouring of $G$. A word $w \in A_{n}^{*}$ is called a factor of $G$ if $w=f\left(v_{1}\right) f\left(v_{2}\right) \cdots f\left(v_{k}\right)$ for some path $v_{1}, v_{2}, \ldots v_{k}$ in $G$ that contains no repeated vertices. An $n$-colouring of the graph $G$ is called $r$-free if it contains no factors of exponent greater than or equal to $r$. By this definition, the 2 -free colourings of $G$ are exactly the nonrepetitive colourings of $G$, first defined by Alon et al. [3]. Nonrepetive colourings have been widely studied in the last two decades. In particular, Dujmovic et al. [14] recently confirmed what was probably the most important conjecture on nonrepetitive colourings, namely that every planar graph can be nonrepetitively coloured with a bounded number of colours. Their paper also contains an extensive list of references to other work on nonrepetitive colourings and related notions.

To date, most work on $r$-free colourings has concerned the problem of fixing a number $r$ (most commonly $r=2$ ) and determining the minimum number of colours necessary for an $r$-free colouring of a given graph. Ochem and Vaslet [21] introduced a notion of repetition threshold for classes of graphs, which considers the problem the other way around - for a fixed number of colours, find the smallest value of $r$ such that there is an $r$-free colouring of a given graph. Formally, the repetition threshold for $n$ letters on $G$, denoted $\operatorname{RT}(n, G)$, is defined by:

$$
\operatorname{RT}(n, G)=\inf \{r>1 \text { : there is an } r \text {-free } n \text {-colouring of } G\} \text {. }
$$

For a collection of graphs $\mathcal{G}$, the repetition threshold for $\mathcal{G}$ is defined by $\mathrm{RT}(n, \mathcal{G})=\sup _{G \in \mathcal{G}} \operatorname{RT}(n, G)$. Note that the strong circular repetition threshold is equivalent to the repetition threshold $\operatorname{RT}(n, \mathcal{C})$, where $\mathcal{C}$ is the collection of cycles.

Ochem and Vaslet determined all values of $\operatorname{RT}(n, \mathcal{T})$, where $\mathcal{T}$ is the collection of all trees. Lužar, Ochem, and Pinlou [17] determined all values of $\operatorname{RT}(n, \mathcal{C P})$ and $\operatorname{RT}\left(n, \mathcal{C P}_{3}\right)$, where $\mathcal{C P}$ is the collection of all caterpillars, and $\mathcal{C} \mathcal{P}_{3}$ is the collection of all caterpillars of maximum degree 3 . They also gave upper and lower bounds on $\operatorname{RT}\left(n, \mathcal{T}_{3}\right)$, where $\mathcal{T}_{3}$ is the collection of all trees of maximum degree 3.

Ochem and Vaslet 21] also defined a notion of repetition threshold for "sufficiently large subdivisions" of all graphs. For a graph $G$, let $\mathcal{S}(G)$ denote the collection of subdivisions of $G$ (i.e., those graphs obtained from $G$ by a sequence of edge subdivisions). For a collection of graphs $\mathcal{G}$, we define the weak repetition threshold for $\mathcal{G}$, denoted $\operatorname{WRT}(n, \mathcal{G})$, by

$$
\operatorname{WRT}(n, \mathcal{G})=\sup _{G \in \mathcal{G}} \inf _{G_{s} \in \mathcal{S}(G)} \operatorname{RT}\left(n, G_{s}\right) .
$$


The repetition threshold for subdivided graphs, as defined by Ochem and Vaslet, is then equivalent to the weak repetition threshold for the collection $\mathcal{H}$ of all graphs. Ochem and Vaslet proved that

$$
\operatorname{WRT}(n, \mathcal{H})= \begin{cases}7 / 3, & \text { if } n=2 \\ 7 / 4, & \text { if } n=3 \\ 3 / 2, & \text { if } n \geq 4\end{cases}
$$

For all $n \geq 4$, the lower bound $\operatorname{WRT}(n, \mathcal{H}) \geq 3 / 2$ follows from the somewhat trivial fact that any $n$-colouring of a graph with a vertex of degree $n$ must contain a factor of exponent $3 / 2$. This suggests restricting to classes of graphs with bounded maximum degree, as was done for the repetition thresholds of caterpillars and trees [17]. Let $\mathcal{H}_{k}$ denote the collection of graphs with maximum degree $k$. For all $n \geq 2$, it is easy to see that we have

$$
\operatorname{WRT}\left(n, \mathcal{H}_{2}\right)=\mathrm{CRT}_{\mathrm{W}}(n)
$$

since every graph of maximum degree 2 is a disjoint union of paths and cycles. So by Theorem 2, we have $\operatorname{WRT}\left(n, \mathcal{H}_{2}\right)=n /(n-1)$ for all $n \geq 45$. In addition to determining the unknown values of $\operatorname{WRT}\left(n, \mathcal{H}_{2}\right)$, it would be interesting to determine values of $\operatorname{WRT}\left(n, \mathcal{H}_{k}\right)$ for $k \geq 3$.

\section{References}

[1] A. Aberkane and J. D. Currie, There exist binary circular $5 / 2^{+}$power free words of every length, Electron. J. Combin. 11(1) (2004), \#R10.

[2] A. Aberkane and J. D. Currie, Attainable lengths for circular binary words avoiding $k$ powers, Bull. Belg. Math. Soc. Simon Stevin 12(4) (2005), 525-534.

[3] N. Alon, J. Grytczuk, M. Hałuszczak, and O. Riordan, Nonrepetitive Colorings of Graphs, Random Structures Algorithms 21(3-4) (2002), 336-346.

[4] J. Berstel, Axel Thue's papers on repetitions in words: A translation, Publications du LaCIM (Université du Québec à Montréal), vol. 20, 1995. 
[5] A. Carpi, On Dejean's conjecture over large alphabets, Theoret. Comput. Sci. 385(1-3) (2007), 137-151.

[6] J. Cassaigne and J. Karhumäki, Toeplitz words, generalized periodicity and periodically iterated morphisms, European J. Combin. 18 (1997), 497-510.

[7] J. D. Currie and N. Rampersad, Dejean's conjecture holds for $n \geq 27$, RAIRO - Theor. Inform. Appl. 43(4) (2009), 775-778.

[8] J. D. Currie and N. Rampersad, Dejean's conjecture holds for $n \geq 30$, Theoret. Comput. Sci. 410(30-32) (2009), 2885-2888.

[9] J. D. Currie and N. Rampersad, A proof of Dejean's conjecture, Math. Comp. 80(274) (2011), 1063-1070.

[10] J. D. Currie, L. Mol, and N. Rampersad, Circular repetition thresholds on some small alphabets: Last cases of Gorbunova's conjecture, Electron. J. Combin. 26(2) (2018), \#P2.31.

[11] J. D. Currie, L. Mol, and N. Rampersad, The number of threshold words on $n$ letters grows exponentially for every $n \geq 27$, preprint (2019). Available at https://arxiv.org/abs/1911.05779.

[12] J. D. Currie, There are ternary circular square-free words of length $n$ for $n \geq 18$, Electron. J. Combin. 9 (2002), \#N10.

[13] F. Dejean, Sur un théorème de Thue, J. Combin. Theory Ser. A 13 (1972), 90-99.

[14] V. Dujmović, L. Esperet, G. Joret, B. Walczak, and D. R. Wood, Planar graphs have bounded nonrepetitive chromatic number, preprint (2019). Available at https://arxiv.org/abs/1904.05269.

[15] I. A. Gorbunova, Repetition threshold for circular words, Electron. J. Combin. 19(4) (2012), \#P11.

[16] M. Lothaire, Algebraic combinatorics on words, Cambridge University Press, 2002. 
[17] B. Lužar, P. Ochem, and A. Pinlou, On repetition thresholds of caterpillars and trees of bounded degree, Electron. J. Combin. 25(1) (2018), \#P1.61.

[18] M. Mohammad-Noori and J. D. Currie, Dejean's conjecture and Sturmian words, European J. Combin. 28(3) (2007), 876-890.

[19] J. Moulin-Ollagnier, Proof of Dejean's conjecture for alphabets with 5, 6, 7, 8, 9, 10, and 11 letters, Theoret. Comput. Sci. 95(2) (1992), 187-205.

[20] H. Mousavi, Automatic theorem proving in Walnut, preprint (2016). Available at https://arxiv.org/abs/1603.06017.

[21] P. Ochem and E. Vaslet, Repetition thresholds for subdivided graphs and trees, RAIRO - Theoretical Informatics and Applications 46(1) (2012), $123-130$.

[22] J. J. Pansiot, A propos d'une conjecture de F. Dejean sur les répétitions dans les mots, Discrete Appl. Math. 7(3) (1984), 297-311.

[23] M. Rao, Last cases of Dejean's conjecture, Theoret. Comput. Sci. 412(27) (2011), 3010-3018.

[24] A. M. Shur, On the existence of minimal $\beta$-powers, Internat. J. Found. Comput. Sci. 22(7) (2011), 1683-1696. 